\title{
Cluster Based Trustable Target Detection and Tracking Scheme for Wireless Sensor Networks
}

\author{
C. Jehan ${ }^{1 *}$ \\ D. Shalini Punithavathani ${ }^{2}$ \\ ${ }^{1}$ Tamizhan College of Engineering and Technology, Tamilnadu, India \\ ${ }^{2}$ Government college of engineering, Tirunelveli, India \\ * Corresponding author's Email: jehanc2001@gmail.com
}

\begin{abstract}
This article intends to present a Cluster based Trustable Target Detection and Tracking Scheme (CTTDTS) for Wireless Sensor Network (WSN). The entire network area is segregated into numerous equal sized grids and each grid acts as a cluster. All the grids are populated with equal count of nodes. A predominant node is elected for each grid by means of trust degree, which is computed by the Dempster Shafer theory. The predominant node fixes the minimal count of active nodes per grid and controls the activity of the nodes. The predominant node along with the active nodes detects and tracks the target. Besides this, the predominant node alerts the neighborhood clusters through which the target may traverse. The network lifetime is considerably enhanced by employing job cycle scheduling and clustering technique. The experimental results prove the efficacy of the proposed approach in terms of detection accuracy, energy consumption and network lifetime.
\end{abstract}

Keywords: Target tracking; trust; detection accuracy; network lifetime; cluster; Wireless Sensor network.

\section{Introduction}

A Wireless Sensor Network (WSN) is formed by numerous sensor nodes and the task of the sensor nodes vary with respect to the application. Basically, a sensor node can sense the data from the environment process the data to some extent and share the information. The process of information sharing is achieved by the communication between the sensor nodes. Normally, the sensor nodes collect the data and share it with the Base Station (BS). In order to perform these operations, the sensor nodes must avail a reasonable battery backup. Mostly the WSN are deployed in remote areas, where human intervention is very minimal. In such cases, battery recharging or replacement is not possible. Thus, exploiting the available energy in an efficient way is a crucial challenge for WSN.

WSN enjoys wider range of applicable areas such as environment monitoring to detect temperature, healthcare monitoring, sensing battle fields, monitoring weather conditions and target tracking [1-3]. Hence WSN involves many surveillance applications, which intends to track a target. The target can be mobile or immobile. The basic idea of target tracking is that the nodes must be vigil at all times, such that the movement pattern of the target can be detected. However, when the sensor nodes remain active at all times to track the target. This lays a serious impact on the network lifetime, as the sensor nodes consume more energy when tracking the targets in a continuous fashion.

A standard target tracking algorithm involves three significant phases and they are target detection, mobility forecast and communication. Initially, the sensor nodes must be capable of detecting the target by a target detection algorithm. The detected target must be followed or tracked, so as to capture the movement of target. Finally, the framed path must be shared with some entity by communication. The major challenges involved in target tracking are detection accuracy, energy efficiency, network structure and so on.

This article aims to present novel clustered target detection and tracking scheme, which relies on the trust degree of sensor nodes and job cycle 
scheduling. The proposed work conserves as much energy as possible, owing to the incorporation of job cycle scheduling and cluster methodologies. The entire work is compartmentalized into four significant phases and they are network area segregation and cluster building, predominant node selection and recycling and target tracking.

The network area segregation phase aims to segregate the entire network area into multiple grids. This idea shoots up the positive effective features such as maintainability, manageability and simplicity. The cluster building phase tends to establish cluster of nodes with respect to the grids. This way of clustering works well irrespective of the degree of node's mobility. As soon as the cluster is formed, it is necessary to pick the most appropriate node to play the role of the cluster head. This node has power over its constituent nodes and governs them. The cluster head schedules the job cycle of the nodes. Finally, the target is detected and tracked by the sensor nodes present in the grids. The work highlights are presented as follows.

- The network area is segregated into several grids, which follows the principle of modularity. This boosts up the manageability and maintenance of nodes.

- The clusters are formed by taking the grids into account, which makes the happening of cluster formation in a streak.

- The mobility of nodes has nothing to play with the formation of clusters. This is because, the clusters are formed with respect to the location of the nodes.

- The cluster head is elected by considering the trust degree into account, such that the most suitable node is elected to control the cluster member nodes.

- The cluster head computes the trust degree of its cluster member nodes and stops the operation of nodes, whenever the trust degree is found to be minimal.

- The trust degree computation of a node relies on two neighborhood nodes, which results in a promising value.

- The cluster head is recycled then and there, so as to preserve the energy of the node and to utilize the energy in a balanced manner.

- The energy of the sensor nodes is very well preserved by means of sleep cycle scheduling. This concept allows a certain number of nodes to be in work cycle and the remaining nodes are in sleep mode.
- The target is detected and tracked effectively, as every grid is considered as a cluster. The movement pattern of the target is tracked by the cluster nodes. Whenever a target crosses a cluster, the cluster head alerts the nearby clusters. This increases the tracking accuracy.

The rest of this article is organized as follows. Section 2 is loaded with the review of the literature with respect to target tracking in WSN. Section 3 presents the system model and assumptions of the work. The proposed methodology is elaborated in section 4. The performance of the proposed methodology is analyzed and the experimental results are presented in section 5. The concluding remarks are drawn in section 6 .

\section{Review of literature}

This section intends to present the related works that exist in the literature with respect to target tracking in WSNs.

In [4], a cluster based target tracking technique for WSN is proposed. The target tracking accuracy rate and energy efficiency are claimed to be greater. The main drawback being cited with respect to this work is that the sensor nodes cannot communicate among themselves. In order to overcome this issue, a hybrid algorithm of static and dynamic clustering techniques is presented in [5]. As soon as the nodes are deployed, static clusters are established. Whenever a target is detected, dynamic clusters are formed, such that the nodes can communicate with each other regarding the target. The dynamic clusters are shattered, when the target passes that region. However, the major drawback of this technique is the cost involved in establishing and dismissing dynamic clusters. Additionally, this work consumes more energy, which reduces the lifetime of the network.

An energy efficient target tracking algorithm for WSN is presented in [6], which is resistant against intrusions. This work forms clusters on the go in dynamic fashion. The clusters are formed by taking the movement pattern of the target into account. The nodes which are near by the target compete with each other to play the role of a cluster head. Whenever the nodes detect the target, the Cluster Head Election Window is constructed. This window is based on the energy and the distance from the node to target. The node with maximum energy and minimal distance from the target is chosen as the cluster head. The disadvantages of this 
work include energy inefficiency and poor network lifetime.

In [7], a prediction based clustering algorithm for target tracking is presented. This work involves two major phases such as clustering and tracking. Initially, the clusters are formed by broadcasting messages to the nodes and the cluster head is selected. Whenever the cluster head detects a target in its region, it chooses three nodes from the cluster for sensing purpose. The selected nodes forward the distance of the node from the target to the cluster head. The location of the target is found by these distances and the next possible location of the nodes is predicted. This work involves communication overhead and more energy consumption.

A filter based technique is presented in [8], which intends to minimize the communication between the clusters. Besides this, binary sensors are employed to track the sensors. The binary sensors just work with two bits 0 and 1 , which indicates the presence and absence of the target. The disadvantage of this work is the tracking inaccuracy. Another prediction based target tracking mechanism is proposed in [9]. This work keeps the nodes present in the network border alert, such that the target can be detected effectively. The execution of the location prediction algorithm depends on the mobility speed of the target. This work involves energy inefficiency and thus, minimal network lifetime.

An effort to achieve target accuracy is presented in [10], which utilizes tree based concept. This work predicts the movement of the target even before the arrival of the target. The node which presents nearer to the target is selected as the root node. The root node then forms a tree and computes the mobility speed and location of the target. The construction of trees improves as the speed of the target improves. This results in computation overhead and maximum energy consumption. Besides this, when the movement pattern of the target varies from the predicted movement pattern the performance is degraded.

This issue is addressed in [11] where the dynamic tree is formed for tracking the target. The adaptive tree is executed all at once, when the target's mobility pattern from the predicted one. The tree is then re-established by using TAP or DAB techniques [12]. The major drawback of this technique is the computational complexity. In [13], a Probability based Target Prediction and Sleep Scheduling (PPSS) is presented. This work predicts the movement of the target by means of kinematics and probability theory. However, this work assumes that the target moves in a smooth path and cannot perform well when the direction of the target differs.

An energy efficient target tracking algorithm is presented in [14], which tends to minimize the count of working nodes. The working nodes are selected on the basis of kinematics principle. However, this work keeps more number of nodes in live mode and this idea results in energy wastage. Another work has been proposed in the literature to control the count of working nodes [15]. In this work, the tracking area is reduced so as to have minimal count of nodes in working state. This work is energy efficient and the lifetime of the network is maximized.

Motivated by the above works, this article proposes a clustered trustable target tracking scheme for WSN, which is effective in terms of energy efficiency and tracking accuracy. The energy efficiency is achieved by the incorporation of cluster based approach and job cycle scheduling of sensor nodes. This idea improves the lifetime of the network as well. The target tracking accuracy is attained by proposing a grid based network, which forms clusters by taking the location coordinates into account. Whenever a cluster detects the target, it immediately alerts the nearby clusters, which paves way for better target tracking accuracy.

\section{System Model and Assumptions}

This work deploys numerous motionless sensor nodes in a wide area to detect a mobile target $M_{t}$. The network area $N_{a}$ is subdivided into several square shaped grids $N_{a}=\left\{N_{l}, N_{2}, \ldots N_{n}\right\}$. Each and every grid consists of equal count of sensor nodes. Every grid is considered as a cluster and a cluster head manages the cluster member nodes. The Base Station (BS) is mobile and it reaches each grid for every period of time. The cluster head is selected by means of trust degree being computed. The cluster head is recycled for every period of time, in order to save energy. Additionally, the cluster head is recycled whenever the energy of the node falls below the energy threshold. The cluster member can take two different labels, which are active and passive. The nodes in active state are in job cycle, whereas the nodes in passive state conserve energy by performing no tasks. This work considers two different categories of nodes and they are staff and border line nodes. The staff nodes present inside the cluster and the border line nodes present on the boundary of the cluster. The next section presents 
the assumptions of the proposed work.

\subsection{Assumptions}

Some of the important assumptions of this work are listed below.

- The entire network area is equally subdivided into numerous equal sized grids.

- All the grids are populated with equal count of sensor nodes.

- All the sensor nodes are immobile, whereas the target and the BS are mobile.

- All the sensor nodes are aware of their location information.

- The sensor nodes present in the network can either be cluster heads or cluster members.

- The sensor nodes being present in the cluster can either be staff or border line nodes.

- The cluster head is represented as predominant node and the cluster members are denoted as constituent nodes.

- The constituent nodes can be either in active or passive state.

- The constituent nodes can communicate with the predominant node directly.

- The predominant nodes can communicate with the BS either in a direct fashion or via other predominant nodes.

Thus, the system model and the assumptions are presented. The forthcoming section portrays the detailed description of the proposed target tracking scheme.

\section{Clustered Trustable Target Detection and Tracking Scheme}

This section elaborates the proposed target tracking scheme along with the overview of the work.

\subsection{Overview of the Proposed Approach}

The main goal of this article is to arrive at an effective target tracking scheme for WSN, which is energy conserving and accurate. The goal is achieved by decomposing the work into several subphases such as network area segregation and cluster building, predominant node selection and recycling and target detection and tracking. The network area segregation and the cluster building phase follows the principle of divide and conquer, which offers easy maintenance and control over the nodes.
Besides this, it improves the effectiveness of the work, because all grids are given equal importance. Each grid is populated with equal count of nodes and every grid act as a cluster. The predominant node is chosen by computing the trust degree. The trust degree is calculated by the Dempster shafer theory. The node with the greatest trust degree is elected as the predominant node. The remaining nodes of the cluster are called as the constituent nodes and these nodes work under the surveillance of the predominant node. The overall flow of the proposed approach is presented in fig. 1.

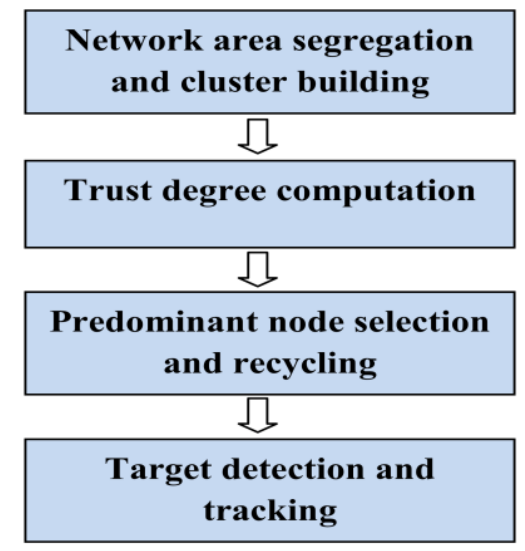

Figure.1 Overall flow of the proposed approach

The main task of the predominant node is to control the state of the nodes, such that the energy of the nodes is preserved. The predominant node must not be overloaded and thus it is recycled for every period of time, so as to maximize the lifetime. However, whenever the current energy of the predominant node falls below the energy threshold, it is recycled immediately. The predominant node can easily detect the target, as it is mobile. As soon as the target is detected, it employs staff and border line nodes to track the target. The predominant node selects one staff and two border line nodes respectively. The predominant node employs three constituent nodes to be in job cycle. This reduces the energy consumption considerably and improves the lifetime of the network. Additionally, the predominant nodes of the neighbouring clusters are alerted, such that the target cannot escape from the detection. The predominant node reports the BS whenever the target crosses the corresponding cluster. This improves the target tracking accuracy of the system.

\subsection{Network Area Segregation and Cluster Building}


The entire network area is segregated into multiple partitions called grids and is represented as $N_{a}=N_{l}, N_{2} \ldots N_{n}$, where $N_{a}$ is the network area and $N_{l}$, $N_{2}, \ldots N_{n}$ are the grids. The network is equally partitioned and the equal count of nodes is distributed in the grids. The reason behind this idea is as follows. When the nodes are randomly distributed across all grids, then certain may be overpopulated with the senor nodes and the remaining grids may contain less nodes or empty. Taking this point into account, this work distributes nodes in uniform fashion.

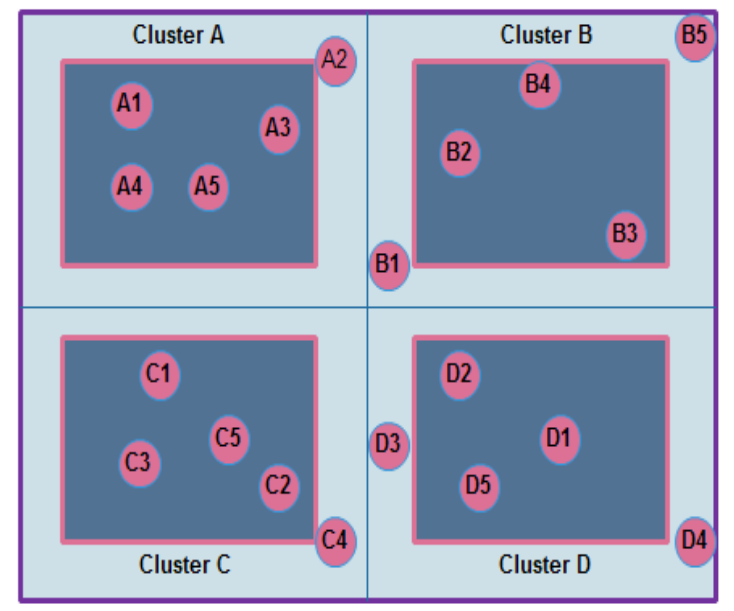

Figure.2 Network area segregation and cluster building

Besides this, it is difficult to globally fix the standard count of nodes to be in job cycle, unless the grids contain equal count of nodes. For instance, consider the work fixes the global count of active nodes as three. However, certain grids may possess only three nodes, where all the nodes must be active. In the next cycle, no nodes would be available to sense. Thus, this work distributes equal count of nodes in all the grids. This phase is illustrated in figure 2 .

In the above figure, the nodes enclosed in pink coloured lines are staff nodes and the nodes present outside the pink box are called borderline nodes. The nodes within each grid form a cluster, which is represented as $N_{n}=\left\{C_{1}, C_{2}, \ldots, C_{n}\right\}$. This idea introduces several advantages to the proposed work and they are as follows. The clusters are formed by considering the nodes of a grid and thus, the clusters can be formed either with the mobile and immobile nodes. The movement of the nodes doesn't affect the clustering process. The overhead involved in forming clusters is also overthrown. Besides this, the node's energy is also preserved as the clusters are formed in a streak. The nodes present in each cluster are represented as $C_{n}=\left\{n_{c 1}, n_{c 2}, \ldots, n_{c n}\right\}$. Thus, the network area is segregated and the clusters are built effectively. The following section presents the procedure for selecting predominant node for the cluster by trust degree computation.

\subsection{Trust Degree Computation}

As soon as the clusters are formed, it is necessary to select the predominant node. This section elaborates the trust degree computation. Initially, the neighbourhood nodes compute the trust degree by means of Dempster Shafer theory. Computation of trust degree is the most important step, as the predominant nodes are selected by the trust degree. The trust metrics being considered by this work are packet transmission rate and energy level. Packet transmission rate is considered as an important trust metric, as it clarifies the node's tendency towards forwarding packets. Certain nodes transmit all the incoming packets, while others may transmit certain packets and the remaining nodes never transmit. As a special case, some nodes repeatedly transmit the same packet, so to waste the available energy and to decrease the lifetime of the network. The nodes that do not transmit packets tend to conserve its energy. However, both selective forwarding and no packet forwarding are serious issues to be handled. Recognizing the importance of packet transmission rate, this work chooses it as a parameter for computing the trust degree.

The energy is the vital parameter of any sensor node, as it decides the lifetime of the sensor node. The goal is achieved only when the energy of the sensor node is reasonable. Thus, energy of a node is also considered as a parameter for computing the trust degree. These two trust metrics are combined together to form the trust degree and is computed by the Dempster Shafer theory [16] as shown below. This work employs Dempster Shafer theory to compute trust degree, as the knowledge of probabilistic theory is not needed. The trust degree is computed for all the nodes by two of its neighbourhood nodes. This way of trust degree computation is reliable.

A sensor node can be declared as trustable, not trustable and either of the two categories. This is represented as given below

$$
\mathrm{x}: \varphi=\left\{T, T^{\prime}\right\}
$$

Equation 1 represents that the node $x$ can either be trustable or non trustable and this equation can be rewritten as follows. 


$$
\begin{gathered}
A=\{T\} \\
A=\left\{T^{\prime}\right\} \\
D=\varphi
\end{gathered}
$$

$A=\{T\}$ indicates that the node is trustable and equation 2 indicates that the sensor node is non trustable. Equation 4 denotes that the sensor node can be trustable or non trustable. Assume that the probability function of trustability and non trustability of $x$ is indicated by $\delta$.

$$
\begin{gathered}
\left\{\begin{array}{c}
\mathrm{k}_{1}(\mathrm{~A})=\delta \\
\mathrm{k}_{1}\left(\mathrm{~A}^{\prime}\right)=0 \\
\mathrm{k}_{1}(\mathrm{D})=1-\delta
\end{array}\right. \\
\left\{\begin{array}{c}
\mathrm{k}_{1}(\mathrm{~A})=0 \\
\mathrm{k}_{1}\left(\mathrm{~A}^{\prime}\right)=\delta \\
\mathrm{k}_{1}(\mathrm{D})=1-\delta
\end{array}\right.
\end{gathered}
$$

Now, the trust degree is computed for all the nodes by the direct neighbours are summed up. The following equations present three different cases, in which both the neighbour nodes declare the corresponding node as trustable. The second case represents that the corresponding node is not trustable. The third case indicates that the corresponding node can either be claimed as trustable or non trustable. All the three cases are presented in the below given equations.

$$
\begin{aligned}
& k_{1}(\mathrm{~A}) \oplus k_{2}(\mathrm{~A})=\frac{1}{q}\left[k_{1}(\mathrm{~A}) k_{2}(\mathrm{~A})+k_{1}(\mathrm{~A}) k_{2}(\mathrm{D})+k_{1}(\mathrm{D}) k_{2}(\mathrm{~A})\right] \\
& k_{1}\left(\mathrm{~A}^{\prime}\right) \oplus k_{2}\left(\mathrm{~A}^{\prime}\right)=\frac{1}{q}\left[k_{1}\left(\mathrm{~A}^{\prime}\right) k_{2}\left(\mathrm{~A}^{\prime}\right)+k_{1}(\mathrm{~A}) k_{2}(\mathrm{D})+k_{1}(\mathrm{D}) k_{2}\left(\mathrm{~A}^{\prime}\right)\right] \\
& k_{1}(\mathrm{D}) \oplus k_{2}(\mathrm{D})=\frac{1}{q}\left[k_{1}(\mathrm{D}) k_{2}(\mathrm{D})\right]
\end{aligned}
$$

Where $q$ is given by

$$
\mathrm{q}=\left\{\begin{array}{c}
k_{1}(\mathrm{~A}) k_{2}(\mathrm{~A})+k_{1}(\mathrm{~A}) k_{2}(\mathrm{D})+k_{1}(\mathrm{D}) k_{2}(\mathrm{~A})+k_{1}\left(\mathrm{~A}^{\prime}\right) k_{2} \mathrm{~A}^{\prime}(\mathrm{D})(10) \\
\quad+k_{1}\left(\mathrm{~A}^{\prime}\right) k_{2}(\mathrm{D})+k_{1}(\mathrm{D}) k_{2}\left(\mathrm{~A}^{\prime}\right)+k_{1}(\mathrm{D}) k_{2}(\mathrm{D})
\end{array}\right.
$$

The equations from 7 to 9 represent all the three cases with respect to the reliability of the sensor node. The end product of these equations ranges between 0 and 1 , where the value 0 denotes that the sensor node is not trustable. On the contrary, the value 1 indicates that the sensor node is trustable. The value of 0.5 indicates that the sensor node is partly trustable. By this way, the trust degree is computed.

\subsection{Predominant Node Selection and Recycling}

Predominant node selection is the most significant process, as it is a challenging task to select the most appropriate node from the group of nodes. The predominant node must be highly eligible to manage and control the activities of the International Journal of Intelligent Engineering and Systems, Vol.9, No.4, 2016 constituent nodes. Additionally, the predominant node must be vigilant against the arrival of the moving target. However, this work can easily figure out the moving target, as all the participating nodes are static. Another important task of the predominant node is to set the state of the nodes.

As soon as the trust degree is computed, the node with greatest trust degree is chosen as the predominant node. The predominant node then selects the top three nodes with greater trust degree to be in active nodes. The remaining nodes are allowed to be in passive state. This work sets the minimum count of active nodes as three. This work possesses staff and border line nodes.

The predominant nodes enforce a constraint that out of three active nodes, two nodes must be staff nodes and a single node must be a border line node. Besides this, the predominant node blocks the node when the trust degree falls below the threshold 0.3 . The trust degree threshold 0.3 is chosen by trial and error method. Dempster Shafer theory is utilized to compute the trust degree. Some of the noteworthy tasks of the predominant nodes are listed below.

- The predominant node is responsible for maintaining the state of the node, which can be active or passive.

- The predominant node appoints the node to be in active or passive state.

- The predominant node has to block the node, if the trust degree of the nodes goes below the trust degree threshold.

- The predominant node alerts the constituent nodes, as soon as it detects the moving target.

- A packet containing the location of the moving target along with the timestamp is forwarded by the predominant node to the $B S$.

- Whenever a target is detected, the predominant nodes alert the neighborhood clusters so as to ensure accurate target tracking.

The predominant node must be recycled then and there, such that the energy of the predominant node will not be over drained. For every two minutes, the predominant node is recycled. However, when the energy of the node drops below 0.3 within two minutes, then the node is immediately recycled. Initially, all the nodes in the network are fully energized and the energy value is represented as 1 .

\subsection{Target Detection and Tracking}


The major objective of the predominant node is to detect the target and to track the target by means of the constituent nodes. In order to conserve the energy, three nodes are selected from each cluster by the predominant node. Out of three active nodes, two nodes are staff nodes and one node must be the borderline node. The borderline node is present on the boundary of the grid, such that there are chances that the target may pass through or near by the borderline node. The staff and the borderline nodes are recycled for every two minutes. However, when the energy level of the active nodes reaches below the energy threshold, then the nodes are immediately recycled.

Initially, the predominant node along with the active nodes remains active in all the grids. The target can easily be detected by the predominant node, as the target is mobile. Whenever a moving object reaches a cluster, two staff and a borderline node act immediately in order to track the target. As the network area is separated into grids, the midpoint of the boundary is noted as $u, v$ along $x$ and $y$ axis. With this measurement, the active nodes measure the distance between themselves and the target. The computed distance is then forwarded to the predominant node by all the three nodes. The forwarded packet is named as $t t_{-} p k t$ consists of the distance and the timestamp. The predominant node verifies the $t t \_p t$ and picks the packet with recent information and reports the same to the $B S$. Besides this, it alerts the nearby cluster, such that the target detection accuracy is achieved. This concept is illustrated in the figure 3 .

The figure 3 demonstrates that when the cluster $D$ detects the target, the active nodes compute the distance between themselves and the target and forwards the $t$ t_pkt along with the timestamp to the predominant node. Thus, the predominant node receives three $t$ _p $p t s$, out of which the predominant node selects the most recent packet and reports the $B S$. In addition to this, the neighborhood predominant nodes of clusters $C, G$ and $H$ are alerted for the arrival of the target. As predicted, the target enters $H$ and then to $G$. In this situation, the predominant nodes of clusters $B, F, J, K$ and $L$ are alerted and the target passes through $J$. At this juncture, the predominant nodes of $E, I, M, N$ and $O$ is measured. Thus, the target is tracked from the beginning till the end without any hassles. Whenever the target leaves a cluster, the recent update about the target is reported to the $B S$. This improves the detection accuracy and the energy consumption is considerably reduced.

The predominant node alerts only the neighborhood clusters. This makes sense that the predominant nodes of the clusters, which the target cannot reach, are not alerted. This idea minimizes the tracking area and saves energy. This energy conservation maximizes the lifetime of the network. The overall algorithm for the proposed approach is presented in next page.

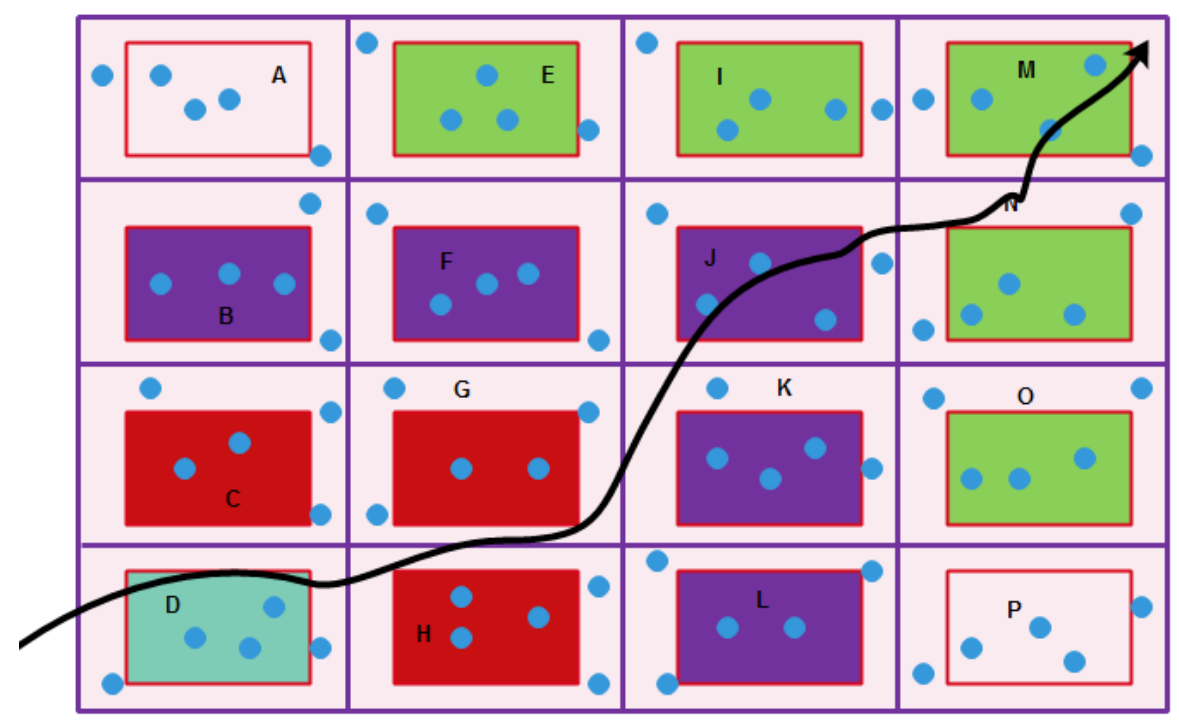

Figure.3 Target detection and tracking CTTTDS - Algorithm 


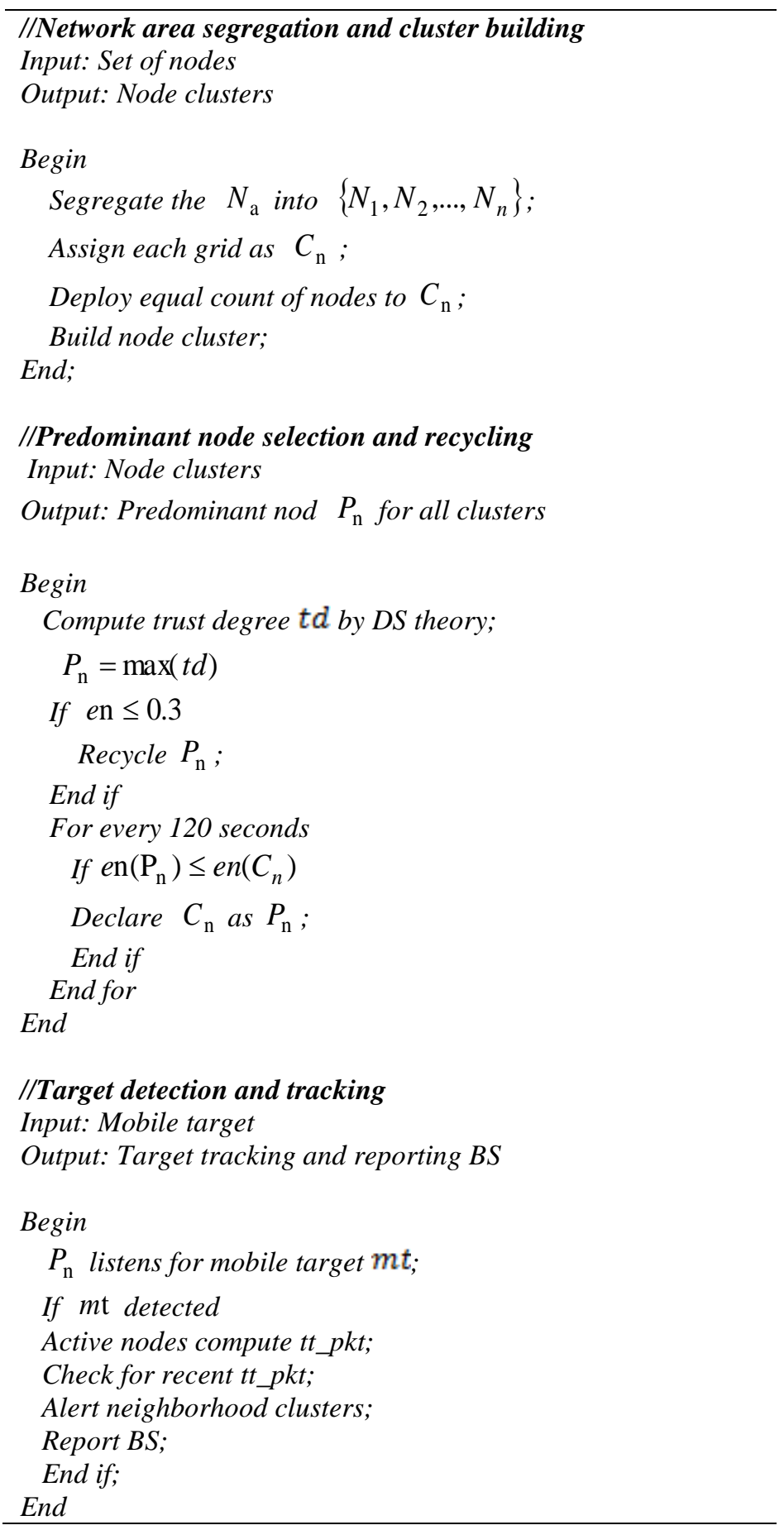

\section{Result and Discussion}

The potentiality of the proposed work is tested with the experimental setup of 100 by 100 squaremetre. This network area is segregated into several 10 by 10 squaremetre grids. The count of nodes being deployed is varied from 500 to 2000 and the count of active nodes is changed between three and ten, in order to evaluate the performance of the proposed approach. Initially, all the nodes are provided with the energy of 60 joules. The energy consumption, network lifetime and the tracking accuracy is measured and compared against PPSS [13] and MCTA [14].

\subsection{Average Energy Consumption Analysis}

An efficient target tracking system must consume lesser energy, as the energy consumption has serious impact over the lifetime of the network. 


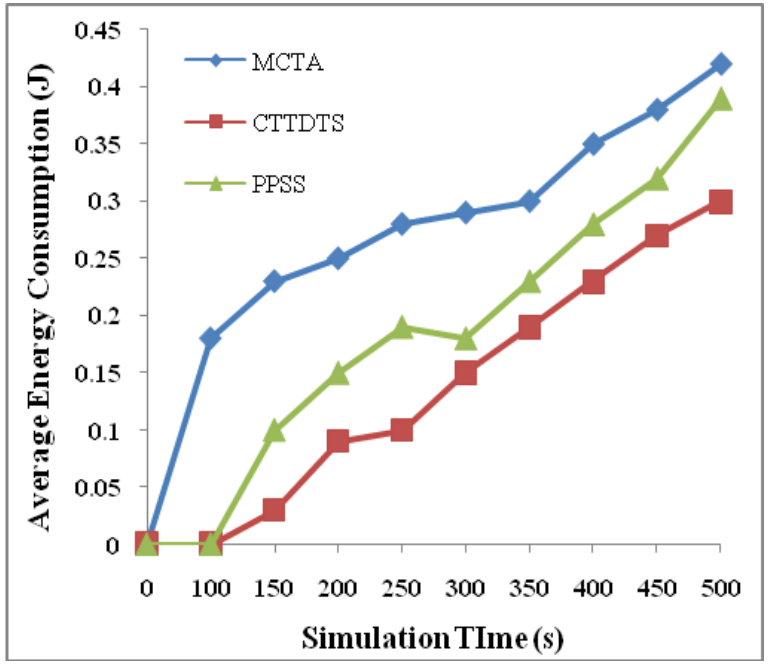

Figure.4 Energy consumption analysis

The average energy consumption of the proposed approach is tested and compared with the existing techniques. The experimental results of energy consumption analysis are presented in fig.4. The energy consumption of the proposed approach is found to be minimal, as it incorporates cluster based approach and schedules the job cycle of the nodes. The active nodes per grid are limited to three, out of which two are staff nodes and a node is a borderline node. The MCTA consumes more energy, though it schedules the nodes. This is because, it exploits more number of awakened nodes, which results in more energy consumption. PPSS does not schedule nodes and it utilizes kinematics concept for predicting the movement of the target, which consumes more energy.

\subsection{Network lifetime Analysis with Regard to Active Nodes}

The lifetime of the proposed approach (CTTDTS) is evaluated by varying the count of active nodes as 2, 5, 7 and 10. It is observed that the lesser number of active nodes show maximum lifetime. On the contrary, more number of active nodes minimizes the lifetime of the network. The experimental results are shown in the fig.5. From the experimental results, it is evident that the count of active node has significant impact over the lifespan of the network. When the active nodes are set as three, 1928 nodes are alive. The count of live nodes falls, as the count of active nodes increases. Finally, when the number of active nodes is set as 10 , the count of live nodes is diminished to 1186 at 500th second.

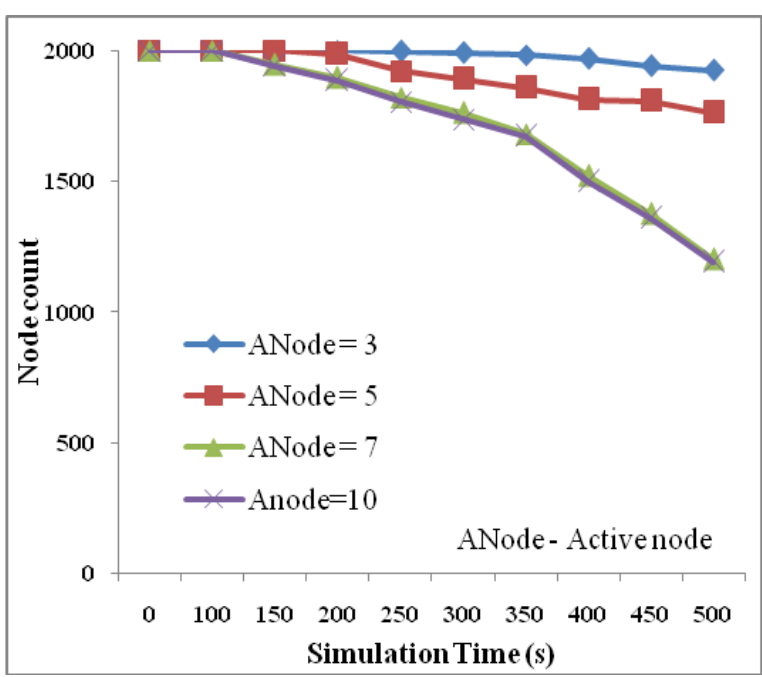

Figure.5 Network lifetime analysis by varying active nodes

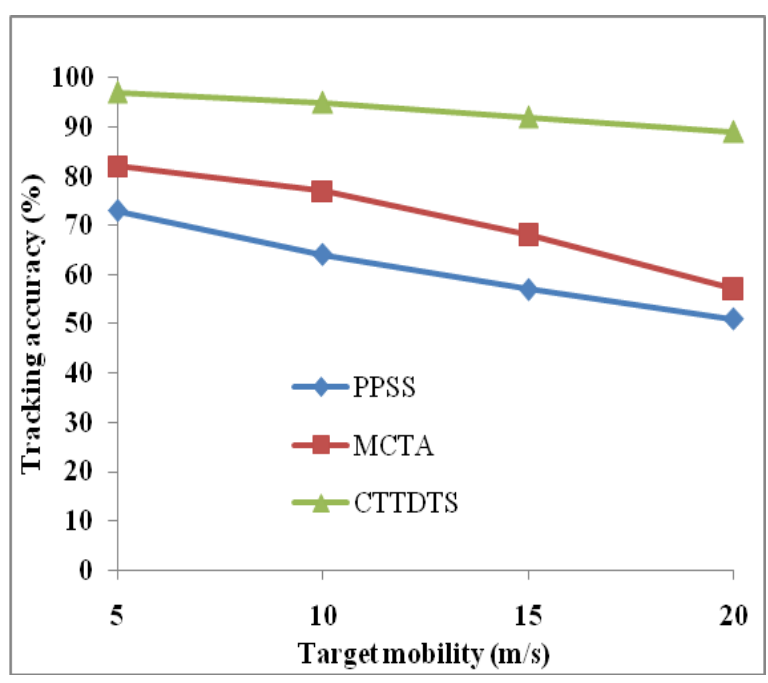

Figure.6 Tracking accuracy analysis

\subsection{Target Detection Accuracy}

The main objective of any target tracking system is to arrive at reasonable target detection accuracy. The purpose of any target tracking system is fulfilled, when the target is perfectly tracked. The detection accuracy of the work is checked by varying the mobility of the target. This section compares the detection accuracy of the proposed approach with the existing approaches and the experimental results are presented in the fig.6. From the experimental analysis, it is proven that the tracking accuracy of the proposed approach is far better than the analogous techniques. The performance of PPSS is poor, as it cannot determine the curved trajectory of the target. MCTA performs better than PPSS but not more than CTTDTS.

The CTTDTS performs well even when the 
target mobility is varied. The reason is that the predominant node alerts the nearby cluster, through which the target may traverse. Besides this, the network area segregation is another important factor for better tracking accuracy, as it covers all the area. Thus, the main objective to enhance the target tracking accuracy and network lifetime is attained by the proposed work.

\section{Conclusion}

In this paper, we have presented a Cluster based Trustable Target Detection and Tracking Scheme (CTTDTS) for WSN. The entire work was decomposed into three major phases such as network area segregation and cluster building, predominant node selection and recycling and target detection and tracking. The initial phase was divided the whole network area into equal sized grids and the clusters are built. The predominant node of all the clusters was selected by the trust degree, which was computed by the Dempster Shafer theory. The targets were tracked by the active nodes and it was forwarded the $t t_{-} p k t$ to the predominant node. The tt_pkt contains the distance between the target and the corresponding node along with the timestamp. The predominant node was selected the recent $t t_{-} p k t$ and reports the BS. Additionally, the predominant node alerts the neighboring clusters such that no target was missed. In future, this work can be enhanced by selecting the active nodes with the help of optimal algorithms.

\section{Reference}

[1] H. Chan and A. Perrig, "Security and privacy in sensor networks", IEEE Computer, Vol. 36, No. 10, pp. 103-105, 2003.

[2] Y.M. Huang, M.Y. Hsieh, H.C. Chao, S.H. Hung and J.H. Park, "Pervasive, secure access to a hierarchical-based healthcare monitoring architecture in wireless heterogeneous sensor networks", IEEE Journal on Selected Areas of Communications, Vol. 27, No. 4, pp. 400-411, 2009.

[3] I. F. Akyildiz, W. Su and Y. Sankarasubramanian, "Wireless Sensor Networks: a survey". Computer Networks Journal, Vol. 38, No. 4, pp. 393-422, 2002.

[4] K. A. Darabkh, S. S. Ismail, M. Al-Shurman, I. F. Jafar, E. Alkhader, and M. F. Al-Mistarihi, "Performance evaluation of selective and adaptive heads clustering algorithms over wireless sensor networks," Journal of Network \& Computer Applications, Vol. 35, No. 6, pp. 2068-2080, 2012.

[5] Z. Wang, W. Lou, Z. Wang, J. Ma and H. Chen, "A hybrid cluster-based target tracking protocol for wireless sensor networks," International Journal of Distributed Sensor Networks, 2013.

[6] J. I. Kong, J. W. Kim, and D.S. Eom, “Energy-aware distributed clustering algorithm for improving network performance in WSNs", International Journal of Distributed Sensor Networks, 2014.

[7] F. Deldar and M. H. Yaghmaee, "Designing a prediction based clustering algorithm for target tracking in wireless sensor networks," in Proceedings of the International Symposium on Computer Networks and Distributed Systems (CNDS '11), IEEE, Tehran, Iran, pp. 199-203, 2011.

[8] J. Teng, H. Snoussi, and C. Richard, "Decentralized variational filtering for target tracking in binary sensor networks," IEEE Transactions on Mobile Computing, Vol. 9, No. 10, pp. 1465-1477, 2010.

[9] Y. Shen, K. T. Kim, J. C. Park, and H. Y. Youn, "Object tracking based on the prediction of trajectory in wireless sensor networks," in Proceedings of the IEEE 10th International Conference on High Performance Computing and Communications \& IEEE International Conference on Embedded and Ubiquitous Computing (HPCC-EUC'13), Zhangjiajie, China, pp. 2317-2324, 2013.

[10] A. Alaybeyoglu, A. Kantarci, and K. Erciyes, "A dynamic look ahead tree based tracking algorithm for wireless sensor networks using particle filtering technique," Computers and Electrical Engineering, Vol. 40, No. 2, pp. 374-383, 2014.

[11] M. X. Chen, C. C. Hu, and W. Y. Weng, "Dynamic object tracking tree in wireless sensor network," EURASIP Journal on Wireless Communications and Networking, Vol. 2010, No. 1, 2010.

[12] H. T. Kung and D. Vlah, "Efficient location tracking using sensor networks," in Proceedings of the IEEE Wireless Communications and Networking Conference (WCNC '03), New Orleans, La, USA, Vol. 3, pp. 1954-1961, 2003.

[13] B. Jiang, B. Ravindran, and H. Cho, "Probabilitybased prediction and sleep scheduling for energyefficient target tracking in sensor networks," IEEE Transactions on Mobile Computing, Vol.12, No. 4, pp. 735-747, 2013.

[14] J. Jeong, T. Hwang, T. He, and D. Du, "MCTA: target tracking algorithm based on minimal contour in wireless sensor networks," IEEE International Conference on Computer Communications (INFOCOM '07), pp. 2371-2375, 2007.

[15] A. A. U. Rahman, M. Naznin, and M. A. I. Mollah, "Energy efficient multiple targets tracking using target kinematics in wireless sensor networks," International Conference on Sensor Technologies and Applications (SENSORCOMM'10), Venice, Italy, pp. 275-280, 2010.

[16] A. P. Dempster, "Upper and lower probabilities induced by a multi-valued mapping". The Annals of Mathematical Statistics, Vol. 38, No. 2, pp. 325-339, 1967. 